\title{
Risk Factors for Psychosis in Parkinson's Disease
}

\author{
Matthew J Barrett \\ University of Virginia, Department of Neurology, Charlottesville, VA, US
}

$\mathrm{P}$

sychosis is a characteristic neuropsychiatric symptom of Parkinson's disease (PD) that is common and associated with worse outcomes. The purpose of this article is to review identified risk factors for visual hallucinations in PD, the most common manifestation of psychosis. With the possible exception of dopamine agonists, antiparkinsonian medications are only considered modifiers of psychosis in PD. Dementia in PD has consistently been shown to be associated with psychosis, and executive dysfunction and impairment in visual processing appear to play a role in its pathogenesis. The association of psychosis with disorders of sleep-wake dysregulation and autonomic dysfunction supports the involvement of brainstem dysfunction in PD psychosis. Despite many studies evaluating genetic risk factors for hallucinations, GBA mutations are the only variants consistently reported to be associated with an increased risk of hallucinations in PD. Lastly, psychosis in PD is associated with a more severe disease burden, both related and unrelated to PD pathology. Any explanatory model of psychosis in PD must incorporate pharmacological, neuroanatomic, pathological, and genetic factors before there can be a complete understanding of this common and disabling neuropsychiatric symptom.

\section{Keywords}

Parkinson's disease, psychosis, hallucinations, risk factor, dementia, rapid eye movement (REM) sleep behavior disorder

Disclosure: Matthew J Barrett has received grant support from the Department of Defense and the commonwealth of Virginia's Alzheimer's and Related Diseases Research Award Fund and has served as site primary investigator for clinical trials funded by the National Institutes of Health, Azevan, Axovant, and Merck. No funding was received in the publication of this article.

Authorship: All named authors meet the International Committee of Medical Journal Editors (ICMJE) criteria for authorship of this manuscript, take responsibility for the integrity of the work as a whole, and have given final approval to the version to be published.

open Access: This article is published under the Creative Commons Attribution Noncommercial License, which permits any non-commercial use, distribution, adaptation and reproduction provided the original author(s) and source are given appropriate credit.

Received: May 22, 2017

Accepted: July 19, 2017

Citation: US Neurology, 2017;13(2):78-81

Corresponding Author: Matthew J Barrett, University of Virginia, Department of Neurology, PO Box 800394 , Charlottesville, VA 22908, US. E: mjbarrett@virginia.edu
In addition to its classic motor features, Parkinson's disease (PD) is associated with neuropsychiatric symptoms, autonomic symptoms, and disorders of sleep and wakefulness. Psychosis is a characteristic neuropsychiatric symptom of PD, most commonly manifesting as visual hallucinations. Other manifestations of psychosis in PD include minor hallucinations (visual illusions, sense of presence, and passage hallucinations), hallucinations in non-visual sensory modalities, and delusions. ${ }^{\text {S Studies }}$ of psychosis in PD almost always include assessment of visual hallucinations with other psychotic symptoms variably assessed.

Hallucinations are common in PD, occurring in 74\% of PD patients after 20 years of disease. ${ }^{2}$ Minor hallucinations may be present at the time of diagnosis and even precede motor symptoms. Pagonabarraga et al. reported that $42 \%$ of de novo PD patients had minor hallucinations, and $33 \%$ had minor hallucinations prior to PD motor symptom onset. ${ }^{3}$ In addition to being common, psychosis is also associated with worse outcomes: increased institutionalization, ${ }^{4}$ decreased quality of life, ${ }^{5}$ and increased mortality. ${ }^{6}$

Considering its prevalence and association with worse outcomes, many studies have sought to identify risk factors for psychosis, specifically visual hallucinations. These efforts have contributed to an improved understanding of this disabling neuropsychiatric symptom. Some risk factors, i.e. cognitive impairment and rapid eye movement (REM) sleep behavior disorder (RBD), may be integral to the mechanism by which psychotic symptoms emerge and have contributed to development of explanatory models of psychotic symptoms in PD. Other risk factors likely represent correlations arising from concomitant pathology in areas not involved in psychosis but occurring at a similar disease stage. Lastly, multiple studies have helped to clarify the relationship between antiparkinsonian medications, especially dopaminergic medications, and psychosis in PD. The purpose of this article is to review risk factors for visual hallucinations in PD that have been replicated in multiple studies.

\section{Medications}

Clinical observation supports a role for dopaminergic medications in PD psychosis: psychotic symptoms may worsen after increasing or adding dopaminergic medications and may improve when these medications are reduced. However, most studies evaluating the role of dopaminergic medications in PD psychosis have shown that antiparkinsonian medication usage is not associated with psychosis. ${ }^{7-12}$ In univariate analyses, Fenelon et al. found that levodopa dose was significantly 
higher in those with visual hallucinations than those without, ${ }^{7}$ and Gibson et al. found that levodopa equivalent daily dosage (LED) was also higher in those with visual hallucinations. ${ }^{12}$ However, neither study found levodopa or LED, respectively, to be an independent predictor of visual hallucinations in multivariate regression models. Contrary to these studies, a 12-year study found that higher LED was associated with increased future risk of psychosis. ${ }^{13}$ The overall evidence coupled with clinical experience has led dopaminergic therapies to be considered modifiers of psychosis in PD. ${ }^{1}$ Dopamine agonists may be an exception to this. Compared with levodopa, the risk of hallucinations is greater with dopamine agonists, ${ }^{14}$ and dopamine agonists increase the risk of psychosis in PD independent of other factors. ${ }^{15,16}$ Other medications, such as anticholinergic drugs, ${ }^{17}$ and medical illness including delirium may elicit or worsen psychosis in PD. For this reason, evaluation of metabolic or pharmacologic causes of psychosis in PD is recommended as the initial treatment step for psychosis in PD. ${ }^{18}$

\section{Cognitive and visual impairment}

Cognitive impairment is one of the most often reported factors associated with psychosis in PD. Psychosis is consistently more common in PD patients with dementia, ${ }^{7,9,11,19-21}$ and cognitive impairment is a risk factor for later developing hallucinations. ${ }^{9,22,23}$ Based on this, many studies have evaluated whether certain cognitive domains are preferentially impaired in PD patients with visual hallucinations. Executive impairment is most consistently reported to be impaired in psychotic patients with $\mathrm{PD}_{1}^{24,25}$ even in those without dementia. ${ }^{26,27}$ It should be noted that these studies used different measures and assessed different aspects of executive dysfunction. Because executive impairment is the cognitive domain most consistently shown to be impaired in PD patients with hallucinations, it is incorporated in most explanatory models. One of these holds that hallucinations in PD arise from a deficit in reality monitoring due to frontal lobe dysfunction..$^{28}$

Due to the primarily visual nature of psychotic symptoms in PD, many tasks of visuospatial function have been assessed in PD psychosis. Overall, studies have found greater impairment in visuospatial and visuoperceptual function in PD patients with hallucinations. ${ }^{24,29-33}$ In addition to visual processing impairment, PD patients with visual hallucinations have reduced visual acuity compared with those without psychosis. 10,34 Altogether, impairments in visual acuity and visual processing in PD with hallucinations indicate that visual deficits contribute to the generation of visual hallucinations.

There are conflicting results regarding whether tests of attention, memory, and language are associated with psychosis in PD. ${ }^{35}$ Explanatory models have sought to integrate deficits in executive function and visuospatial function into a cohesive explanatory model. ${ }^{28} \mathrm{It}$ is evident that the cognitive deficits associated with psychosis are widely distributed and it is likely that the interplay of these contribute to the emergence of visual hallucinations.

\section{Sleep disorders and autonomic dysfunction}

Psychosis is associated with a range of sleep disturbances in PD. A number of studies found an association between visual hallucinations and RBD, 16,21,36,37 and in one study RBD was a predictor of future psychosis. ${ }^{13}$ Additionally, compared to those without hallucinations, PD subjects with hallucinations are more likely to have daytime somnolence, ${ }^{7}$ severe sleep fragmentation, ${ }^{37,38}$ and vivid dreams. ${ }^{37,39}$ Recognition of sleepwake dysregulation in PD with hallucinations led to the hypothesis that hallucinations were caused by intrusion of dream imagery into wakefulness. ${ }^{40}$ One criticism of this explanatory model is that the content of dreams and dream imagery differ from the visual hallucinations reported in PD. Another explanation for the association of hallucinations and sleep-wake dysregulation is that both are caused by overlapping or contiguous areas of brainstem dysfunction. ${ }^{24}$ This idea seems to be supported by the finding that autonomic dysfunction is also associated with hallucinations in PD. 16,22,24 In a 5-year longitudinal study, Zhu et al. found that autonomic dysfunction was an independent risk factor for future hallucinations. ${ }^{41}$ Autonomic dysfunction in PD is linked to pathology of the dorsal motor nucleus of the vagus and other brainstem nuclei in addition to dysfunction of peripheral autonomic nerves. ${ }^{42}$ Therefore, the association of RBD and autonomic dysfunction with hallucinations supports a role for brainstem pathology in the pathogenesis of psychosis in PD. This is supported by a previous study that showed reduced volume of the pedunculopontine nucleus in PD subjects with hallucinations. ${ }^{43}$

\section{Age, duration of disease, and disease severity}

considering that psychosis in PD is a feature associated with advanced disease, it is not surprising that longer disease duration is a risk factor for hallucinations. ${ }^{7,11,12,15}$ Studies have also found that greater disease severity, as measured by the Activities of Daily Living subscale of the Unified Parkinson Disease Rating Scale (UPDRS Part II), is an independent risk factor for hallucinations in PD. 8,10,12,13 Papapetropoulos et al. found that the total UPDRS score was an independent predictor of visual hallucinations in PD. "1 There also appears to be an effect of age, independent of duration of disease. Studies have shown that older age of onset is associated with increased risk of hallucinations. ${ }^{13,23,41}$ In addition to its association with increased risk of hallucinations, advanced age at onset is an independent risk factor for more rapid motor decline and greater disability as measured by the UPDRS Part II and Schwab and England Scale. ${ }^{44}$ Potential explanations for the association between age and psychosis in PD are the age-related increase in visual impairment, burden of comorbid illness, and presence of other cerebral pathologies associated with age, i.e. Alzheimer's disease and cerebrovascular disease. The finding by Biglan et al. that the risk of hallucinations was increased in those with greater comorbid disease burden supports the idea that increasing comorbidities with age are partly responsible for the increased risk of psychosis in older PD patients. ${ }^{23}$ In summary, psychosis in PD is associated with a more advanced and severe disease burden, both related and unrelated to PD pathology, as evidenced by its association with greater disease duration and increased age of onset.

\section{Depression and anxiety}

Some studies have reported an association between visual hallucinations

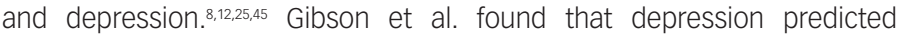
future visual hallucinations. ${ }^{12}$ While it has received less attention than depression, two prior studies reported that anxiety was associated with visual hallucinations. ${ }^{12,46}$ Whether the association between depression and visual hallucinations is merely correlative or should imply that depression increases risk of psychotic symptoms in PD akin to depression with psychotic features, requires further study.

\section{Genetic variants}

Numerous studies have evaluated genetic risk factors for psychosis in PD. They have focused on candidate genes related to neurotransmitters and those implicated in cognitive impairment in PD. Investigations of the 
dopamine receptor and dopamine transporter genes led to inconsistent results. ${ }^{47-49}$ Investigation of genes in the serotonin system showed no association between specific polymorphisms and visual hallucinations in PD. ${ }^{50}$ For the cholecystokinin system, visual hallucinations have been associated with a specific cholecystokinin allele ${ }^{51,52}$ and with a combination of cholecystokinin and cholecystokinin receptor polymorphisms, ${ }^{51,53}$ but a more recent study found no association between cholecystokinin genes and hallucinations. ${ }^{54}$

PD patients with heterozygous GBA (glucosidase, beta, acid) mutations have greater rates of psychotic symptoms. ${ }^{55-57}$ This finding is thought to be related to more accelerated Lewy body pathology in PD patients with GBA mutations. ${ }^{58}$ Indeed, psychosis in PD has been associated with greater cortical Lewy body pathology.24,59,60 Two studies found an association between APOE (Apolipoprotein E) e4 allele and hallucinations, ${ }^{61,62}$ while other studies did not. ${ }^{77,63,64}$ The MAPT (Microtubule-associated protein tau) $\mathrm{H1} / \mathrm{H} 1$ genotype was associated with hallucinations in one study, ${ }^{63}$ while a cross-sectional study found no association between MAPT H1/H1 and psychotic symptoms. ${ }^{64}$ With the exception of GBA mutations, no other genetic variants have consistently been found to be associated with increased risk of hallucinations. Conflicting results may stem from crosssectional study designs or the variable and incomplete assessment of psychotic symptoms. Longitudinal studies evaluating genetic predictors would have an advantage over older studies and would perhaps resolve some of the previous discrepancies.

\section{Conclusion}

Studies evaluating risk factors for psychosis in PD have identified a number of risk factors that provide insight into its underlying mechanism. Psychosis in PD is a manifestation of advanced disease, both related and unrelated to PD pathology. Its association with GBA mutations underscores its relationship with cortical Lewy bodies. Indeed, visual hallucinations are a defining characteristic of dementia with Lewy bodies. The association of psychosis with RBD, other disorders of sleep-wake dysregulation, and autonomic dysfunction support the involvement of brainstem dysfunction in psychosis in PD. ${ }^{24}$ Greater cognitive impairment, specifically in the areas of executive function and visual processing, implicate specific regional cerebral dysfunction in the generation and impaired suppression of psychotic symptoms. Lastly, antiparkinsonian medications modify the presence of psychosis in PD by facilitating psychotic symptoms in some patients. Any explanatory model of psychosis in PD must incorporate these genetic, pathological, neuroanatomic, and pharmacological factors before there can be a complete understanding of this common and disabling neuropsychiatric symptom. $\square$
1. Ravina B, Marder K, Fernandez HH, et al., Diagnostic criteria for syychosis in Parkinson's disease: report of an NINDS, NIMH work group, Mov Disord, 2007;22:1061-8.

2. Hely MA, Reid WG, Adena MA, et al., The sydney multicenter study of Parkinson's disease: the inevitability of dementia at 20 years, Mov Disord, 2008:23:837-44.

3. Pagonabarraga J, Martinez-Horta S, Fernandez de Bobadilla R et al., Minor hallucinations occur in drug-naive Parkinson's disease patients, even from the premotor phase, Mov Disord 2016;31:45-52

4. Aarsland D, Larsen JP, Tandberg E, Laake K, Predictors of nursing home placement in Parkinson's disease: a population-based, prospective study, I Am Geriatr Soc, 2000;48:938-42.

5. McKinlay A, Grace RC, Dalrymple-Alford JC, et al., A profile of neuropsychiatric problems and their relationship to quality of life for Parkinson's disease patients without dementia, Parkinsonism Relat Disord, 2008;14:37-42.

6. Goetz CG, Stebbins GT, Mortality and hallucinations in nursing home patients with advanced Parkinson's disease, Neurology, 1995:45:669-71.

7. Fenelon G, Mahieux F, Huon R, Ziegler M, Hallucinations in Parkinson's disease: prevalence, phenomenology and risk factors, Brain, 2000;123(Pt 4):733-45.

8. Aarsland D, Larsen JP, Cummings JL, Laake K, Prevalence and clinical correlates of psychotic symptoms in Parkinson disease: community-based study, Arch Neurol, 1999;56:595-601.

9. Merims D, Shabtai H, Korczyn AD, et al., Antiparkinsonia medication is not a risk factor for the development of hallucinations in Parkinson's disease, I Neural Transm, 2004:111:1447-53.

10. Holroyd S, Currie L, Wooten GF, Prospective study of hallucinations and delusions in Parkinson's disease, J Neurol Neurosurg Psychiatry, 2001;70:734-738

11. Papapetropoulos S, Argyriou AA, Ellul J, Factors associated with drug-induced visual hallucinations in Parkinson's disease, Neurol, 2005;252:1223-8.

12. Gibson G, Mottram PG, Burn DJ, et al., Frequency, prevalence, incidence and risk factors associated with visual hallucinations in a sample of patients with Parkinson's disease: a longitudinal 4-year study, Int J Geriatr Psychiatry, 2013;28:626-31.

13. Forsaa EB, Larsen JP, Wentzel-Larsen T, A 12-year populationbased study of psychosis in Parkinson disease, Arch Neurol, 2010;67:996-1001.

14. Stowe RL, Ives NJ, Clarke C, et al., Dopamine agonist therapy in early Parkinson's disease, Cochrane Database Syst Rev, 2008;2:CD006564

15. Morgante L, Colosimo C, Antonini A, et al., Psychosis associated to Parkinson's disease in the early stages: relevance of cognitive decline and depression, J Neurol Neurosurg Psychiatry, 2012;83:76-82

16. Barrett MJ, Smolkin ME, Flanigan JL, et al., Characteristics, correlates, and assessment of psychosis in Parkinson disease without dementia, Parkinsonism Relat Disord, 2017: Epub ahead of print. DOI: http://dx.doi.org/10.1016/..parkreldis.2017.07.011

17. Goetz CG, Tanner CM, Klawans HL, Pharmacology of hallucinations induced by long-term drug therapy, Am 」 Psychiatry, 1982;139:494-7.
18. Goldman JG, Vaughan CL, Goetz CG, An update expert opinion on management and research strategies in Parkinson's disease psychosis, Expert Opin Pharmacother, 2011;12:2009-24.

19. Aarsland D, Ballard C, Larsen JP, MCKeith I, A comparative study of psychiatric symptoms in dementia with Lewy bodies and Parkinson's disease with and without dementia, Int J Geriatr Psychiatry, 2001;16:528-36

20. Kitayama M, Wada-Isoe K, Nakaso K, et al., Clinical evaluation of Parkinson's disease dementia: association with aging and visual hallucination, Acta Neurol Scand, 2007;116:190-5.

21. Pacchetti $C$, Manni $R$, Zangaglia R, et al., Relationship between hallucinations, delusions, and rapid eye movement sleep behavior disorder in Parkinson's disease, Mov Disord, 2005:20:1439-48

22. Williams DR, Lees AJ, Visual hallucinations in the diagnosis of idiopathic Parkinson's disease: a retrospective autopsy study, Lancet Neurol, 2005;4:605-10.

23. Biglan KM, Holloway RG, Jr, McDermott MP, et al., Risk factors for somnolence, edema, and hallucinations in early Parkinson disease, Neurology, 2007:69:187-95.

24. Gallagher DA, Parkkinen L, O'Sullivan SS, et al., Testing an aetiological model of visual hallucinations in Parkinson's disease Brain, 2011:134:3299-309.

25. Marsh L, Williams JR, Rocco M, et al., Psychiatric comorbidities in patients with Parkinson disease and psychosis, Neurology, 2004;63:293-300

26. Grossi D, Trojano L, Pellecchia MT, et al., Frontal dysfunction contributes to the genesis of hallucinations in non-demented Parkinsonian patients, Int J Geriatr Psychiatry, 2005;20:668-73.

27. Ozer F, Meral $H$, Hanoglu $L$, et al., Cognitive impairment patterns in Parkinson's disease with visual hallucinations, J Clin Neurosci, 2007:14:742-6.

28. Muller AJ, Shine JM, Halliday GM, Lewis SJ, Visual hallucinations in Parkinson's disease: theoretical models, Mov Disord 2014;29:1591-8.

29. Barnes J, Boubert L, Harris J, et al., Reality monitoring and visual hallucinations in Parkinson's disease, Neuropsychologia, 2003:41:565-74

30. Ramirez-Ruiz B, Junque C, Marti MJ, et al., Neuropsychological deficits in Parkinson's disease patients with visual hallucinations, Mov Disord, 2006;21:1483-7.

31. Meppelink AM, Koerts J, Borg M, et al., Visual object recognition and attention in Parkinson's disease patients with visual hallucinations, Mov Disord, 2008;23:1906-12.

32. Koerts J, Borg MA, Meppelink AM, et al., Attentional and perceptual impairments in Parkinson's disease with visua hallucinations, Parkinsonism Relat Disord, 2010;16:270-4.

33. Diederich NJ, Goetz CG, Raman R, et al., Poor visual discrimination and visual hallucinations in Parkinson's disease, Clin Neuropharmacol 1998;21:289-95

34. Matsui H, Udaka F, Tamura A, et al., Impaired visual acuity as a risk factor for visual hallucinations in Parkinson's disease, J Geriat Psychiatry Neurol, 2006;19:36-40.

35. Lenka A, Hegde S, Arumugham SS, Pal PK, Pattern of cognitive impairment in patients with Parkinson's disease and psychosis: a critical review, Parkinsonism Relat Disord 2017;37:11-18.
36. Nomura T, Inoue $\mathrm{Y}$, Mitani $\mathrm{H}$, et al., Visual hallucinations as REM sleep behavior disorders in patients with Parkinson's disease, Mov Disord, 2003;18:812-17.

37. Goetz CG, Ouyang B, Negron A, Stebbins GT, Hallucinations and sleep disorders in PD: ten-year prospective longitudinal study, Neurology, 2010;75:1773-9.

38. Whitehead DL, Davies AD, Playfer JR, Turnbull CJ, Circadian rest-activity rhythm is altered in Parkinson's disease patients with hallucinations, Mov Disord, 2008;23:1137-45

39. Gama RL, de Bruin VM, de Bruin PF, et al., Risk factors for visual hallucinations in patients with Parkinson's disease, Neurol Res, 2015;37:112-16

40. Arnulf I, Bonnet AM, Damier P, et al., Hallucinations, REM sleep, and Parkinson's disease: a medical hypothesis, Neurology, 2000;55:281-8.

41. Zhu K, van Hilten JJ, Putter H, Marinus J, Risk factors for hallucinations in Parkinson's disease: results from a large prospective cohort study, Mov Disord, 2013;28:755-62.

42. Jellinger KA, Neuropathobiology of non-motor symptoms in Parkinson disease, J Neural Transm (Vienna), 2015;122:1429-40.

43. Janzen J, van 't Ent D, Lemstra AW, et al., The pedunculopontine nucleus is related to visual hallucinations in Parkinson's disease: preliminary results of a voxel-based morphometry study, I Neurol, 2012;259:147-54

44. Alves G, Wentzel-Larsen T, Aarsland D, Larsen JP, Progression of motor impairment and disability in Parkinson disease: a population-based study, Neurology, 2005:65:1436-41.

45. Giladi N, Treves TA, Paleacu D, et al., Risk factors for dementia, depression and psychosis in long-standing Parkinson's disease, I Neural Transm (Vienna), 2000;107:59-71.

46. Mosimann UP, Rowan EN, Partington CE, et al., Characteristics of visual hallucinations in Parkinson disease dementia and dementia with Lewy bodies, Am I Geriatr Psychiatry, 2006;14:153-60

47. Goetz CG, Burke PF, Leurgans S, et al., Genetic variation analysis in Parkinson disease patients with and without hallucinations: case-control study, Arch Neurol, 2001:58:209-13.

48. Makoff AJ, Graham JM, Arranz MJ, et al., Association study of dopamine receptor gene polymorphisms with drug-induced hallucinations in patients with idiopathic Parkinson's disease, Pharmacogenetics, 2000;10:43-8.

49. Wang J, Zhao C, Chen B, Liu ZL, Polymorphisms of dopamine receptor and transporter genes and hallucinations in Parkinson's disease, Neurosci Lett, 2004;355:193-6.

50. Kiferle L, Ceravolo R, Petrozzi L, et al., Visual hallucinations in Parkinson's disease are not influenced by polymorphisms of serotonin 5-HT2A receptor and transporter genes, Neurosci Lett, 2007;422:228-31.

51. Wang J, Si YM, Liu ZL, Yu L, Cholecystokinin, cholecystokinin-A receptor and cholecystokinin-B receptor gene polymorphisms in Parkinson's disease, Pharmacogenetics, 2003;13:365-9.

52. Fujii C, Harada S, Ohkoshi N, et al., Association between polymorphism of the cholecystokinin gene and idiopathic Parkinson's disease, Clin Genet, 1999;56:394-9. 
53. Goldman JG, Goetz CG, Berry-Kravis E, et al., Genetic polymorphisms in Parkinson disease subjects with and without hallucinations: an analysis of the cholecystokinin system, Arch Neurol, 2004;61:1280-4.

54. Goldman JG, Marr D, Zhou L, et al., Racial differences may influence the role of cholecystokinin polymorphisms in Parkinson's disease hallucinations, Mov Disord, 2011;26:1781-2.

55. Wang C, Cai Y, Gu Z, et al., Clinical profiles of Parkinson's disease associated with common leucine-rich repeat kinase 2 and glucocerebrosidase genetic variants in chinese individuals, Neurobiol Aging, 2014;35:725.e1-725.e6.

56. Barrett MJ, Shanker VL, Severt WL, et al., Cognitive and antipsychotic medication use in monoallelic GBA-related Parkinson disease, JIMD Rep, 2014;16:31-8.
57. Oeda T, Umemura A, Mori Y, et al., Impact of glucocerebrosidase mutations on motor and nonmotor complications in Parkinson's disease, Neurobiol Aging, 2015;36:3306-13.

58. Poulopoulos M, Levy OA, Alcalay RN, The neuropathology of genetic Parkinson's disease. Mov Disord 2012;27:831-42.

59. Papapetropoulos S, McCorquodale DS, Gonzalez J, et al., Cortical and amygdalar Lewy body burden in Parkinson's disease patients with visual hallucinations, Parkinsonism Relat Disord, 2006;12:253-6

60. Harding AJ, Broe GA, Halliday GM, Visual hallucinations in Lewy body disease relate to lewy bodies in the temporal lobe, Brain 2002;125:391-403.
61. de la Fuente-Fernandez R, Nunez MA, Lopez E, The apolipoprotein E epsilon 4 allele increases the risk of drug-induced hallucinations in Parkinson's disease, Clin Neuropharmacol, 1999;22:226-30.

62. Feldman B, Chapman J, Korczyn AD, Apolipoprotein epsilon4 advances appearance of psychosis in patients with Parkinson's disease, Acta Neurol Scand, 2006;113:14-17.

63. Papapetropoulos S, Farrer MJ, Stone JT, et al., Phenotypic associations of tau and ApoE in Parkinson's disease, NeurosCl Lett, 2007;414:141-4

64. Factor SA, Steenland NK, Higgins DS, et al., Disease-related and genetic correlates of psychotic symptoms in Parkinson's disease, Mov Disord, 2011;26:2190-5. 